\title{
THEORETICAL CALCULATIONS OF AN OSMIUM MOLECULAR SWITCH
}

\author{
FERNANDO MIRANDA-BARRIENTOS, ALVARO MUÑOZ-CASTRO, RAMIRO ARRATIA-PÉREZ, \\ DESMOND MACLEOD CAREY*
}

Universidad Andres Bello, Departamento de Ciencias Quimicas, Relativistic Molecular Physics (ReMoPh) group, República 275, Santiago, Chile

(Received: August 1, 2013 - Accepted: November 21, 2013)

\begin{abstract}
We have investigated the molecular, electronic and optical properties of the $[\text { Os(tpy-py) }]_{2}^{2+}$ complex (tpy-py $=4^{\prime}-(4-$ pyridyl)-2,2':6',2"'-terpyridine) and its protonated derivative $\left[\mathrm{Os}(\mathrm{tpy}-\mathrm{pyH})_{2}\right]^{4+}$ through Density Functional Relativistic calculations including Scalar and Spin Orbit corrections. The molecular geometry of the parent complex is not strongly modified by the protonation at the basic nitrogen atoms of the pyridine moieties of the terpyridine ligands in the complex. On the other hand, the optical properties of these complexes can be controlled by a change in the chemical acid-base environment, converting them into suitable materials to act as molecular switches or $\mathrm{pH}$ sensor devices.
\end{abstract}

\section{INTRODUCTION}

The innovative designs and creative thinking of numerous scientists led to artificial molecular mechanisms being used in device applications. [1] In this sense, molecular Switches comprise an interesting class of artificial molecular machines and can be defined as molecules that can be switched between two or more different states. In a particular case, Photochemical switches are molecular or supramolecular species in which properties or functions can be switched on and off by light. [1,2]

In this context, recently $[3,4]$ the photoluminescence in the far red spectral region and photosensitised generation of singlet oxygen, with associated near-IR emission, of the $\left[\mathrm{Os}(\text { tpy-py) })_{2}\right]^{2+}$ complex (tpy-py= 4'-(pyridin-4yl)-2,2':6',2"- terpyridine) has been reported. Also, the wavelength of the photoluminescence can be modulated through the a chemical acid-base process, where a protonated form of the $\left[\mathrm{Os}(\mathrm{tpy}-\mathrm{py})_{2}\right]^{2+}$ complex, i.e. $\left[\mathrm{Os}(\mathrm{tpy}-\mathrm{pyH})_{2}\right]^{4+}$ emit at larger wavelengths than the parent complex, see Figure 1.

The aim of this article is to be a first theoretical approach to analyse the molecular and electronic structure, as well as the optical properties of the free base and protonated forms of these bis-terpyridine Osmium complexes, employing current relativistic density functional methodologies.

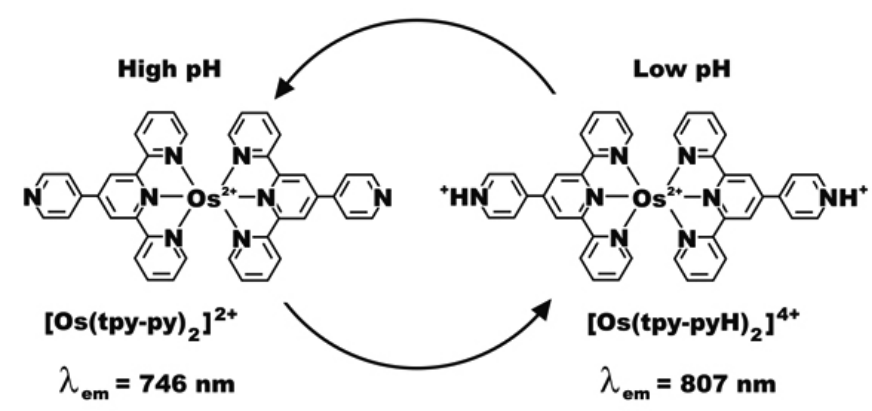

Figure 1: The Osmium Molecular Switch

\section{Computational Details}

The relativistic density functional theory calculations were done using the ADF 2010.01 code [5], incorporating spin-orbit corrections via the twocomponent ZORA Hamiltonian [6,7]. For the calculations we employed triple-z Slater basis set plus polarization function (STO-TZP). For the geometry optimizations we constrained the symmetry as $\mathrm{D}_{2} \mathrm{~d}$ for all the studied complexes. The structures were fully optimized via analytical energy gradient methods implemented by Versluis and Ziegler [8], within the generalized gradient approximation (GGA) of Perdew-Wang (PW91) approximation for the exchange and correlation potential [9]. The Relativistic TD-DFT calculations have been performed on the optimized geometries with the ADF-RESPONSE module [10] which is an extension of the ADF package. The dichloroethane solvent phase was modelled employing the COnductor-like Screening MOdel
(COSMO) [11] as implemented in ADF. The solvent excluding surface was used along with an epsilon value of 10.66 for the dielectric constant of dichloroethane as the solvent. Allinger Atomic radii used were 1.958, 1.700, 1.608 , and $1.350 \AA$ for Os, C, N, and H, respectively.

\section{RESULTS AND DISCUSSION}

The molecular geometries of the titled complexes were constrained to $\mathrm{D}_{2} \mathrm{~d}$ symmetry and optimized at Scalar and SpinOrbit DFT Relativistic level. As can be observed in Table 1, both complexes, $\left[\mathrm{Os}(\text { tpy-py) })_{2}\right]^{2+}$ and $\left[\mathrm{Os}(\text { tpy-pyH })_{2}\right]^{4+}$, exhibit similar molecular structures. The major differences are produced at the pyridine moieties due to the protonation of the nitrogen located at those rings.

Table 1: Selected distances $(\AA)$. Labels according to Scheme 1

\begin{tabular}{|c|c|c|c|c|}
\hline & \multicolumn{2}{|c|}{$\left.[\text { Os(tpy-py })_{2}\right]^{2+}$} & \multicolumn{2}{c|}{$\left.[\text { Os(tpy-pyH })_{2}\right]^{4+}$} \\
\hline & Scalar & Spin Orbit & Scalar & Spin Orbit \\
\hline$d$ Os- $\mathrm{N}_{\mathrm{a}}$ & 2.087 & 2.092 & 2.086 & 2.096 \\
\hline$d \mathrm{Os}_{\mathrm{N}} \mathrm{N}_{\mathrm{b}}$ & 2.003 & 2.008 & 2.005 & 2.009 \\
\hline$d \mathrm{C}_{\mathrm{a}}-\mathrm{N}_{\mathrm{b}}$ & 1.366 & 1.366 & 1.373 & 1.374 \\
\hline$d \mathrm{C}_{\mathrm{a}}-\mathrm{C}_{\mathrm{b}}$ & 1.390 & 1.390 & 1.382 & 1.382 \\
\hline$d \mathrm{C}_{\mathrm{b}}-\mathrm{C}_{\mathrm{c}}$ & 1.407 & 1.408 & 1.426 & 1.426 \\
\hline$d \mathrm{C}_{\mathrm{c}}-\mathrm{C}_{\mathrm{d}}$ & 1.483 & 1.483 & 1.439 & 1.438 \\
\hline$d \mathrm{C}_{\mathrm{d}}-\mathrm{C}_{\mathrm{e}}$ & 1.404 & 1.404 & 1.432 & 1.432 \\
\hline$d \mathrm{C}_{\mathrm{e}}-\mathrm{C}_{\mathrm{f}}$ & 1.394 & 1.394 & 1.365 & 1.366 \\
\hline$d \mathrm{C}_{\mathrm{f}}-\mathrm{N}_{\mathrm{c}}$ & 1.339 & 1.339 & 1.371 & 1.371 \\
\hline
\end{tabular}

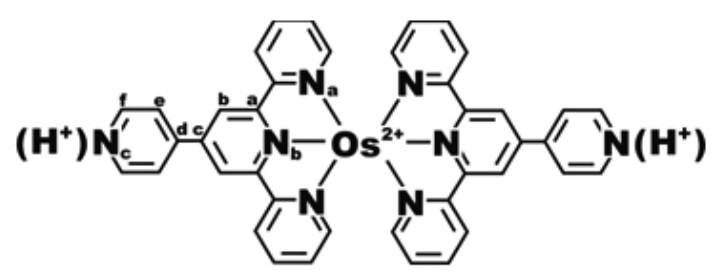

Scheme 1

The electronic structures of the studied complexes are presented in Figure 2. There are just minor changes induced by the inclusion of the Spin Orbit coupling to the theoretical calculations. The inclusion of Spin orbit effects does not affects the size of the HOMS-LUMS gap (HOMS= Highest Occupied Molecular Spinor and LUMS= Lowest Unoccupied Molecular Spinor) in the studied complexes, nevertheless there exist Spin Orbit coupling effects that affect specially the doubly degenerated Molecular Spinors (MS) which expands as $e$ at relativistic Scalar level. The splitting of those $e$ degenerated MS does 


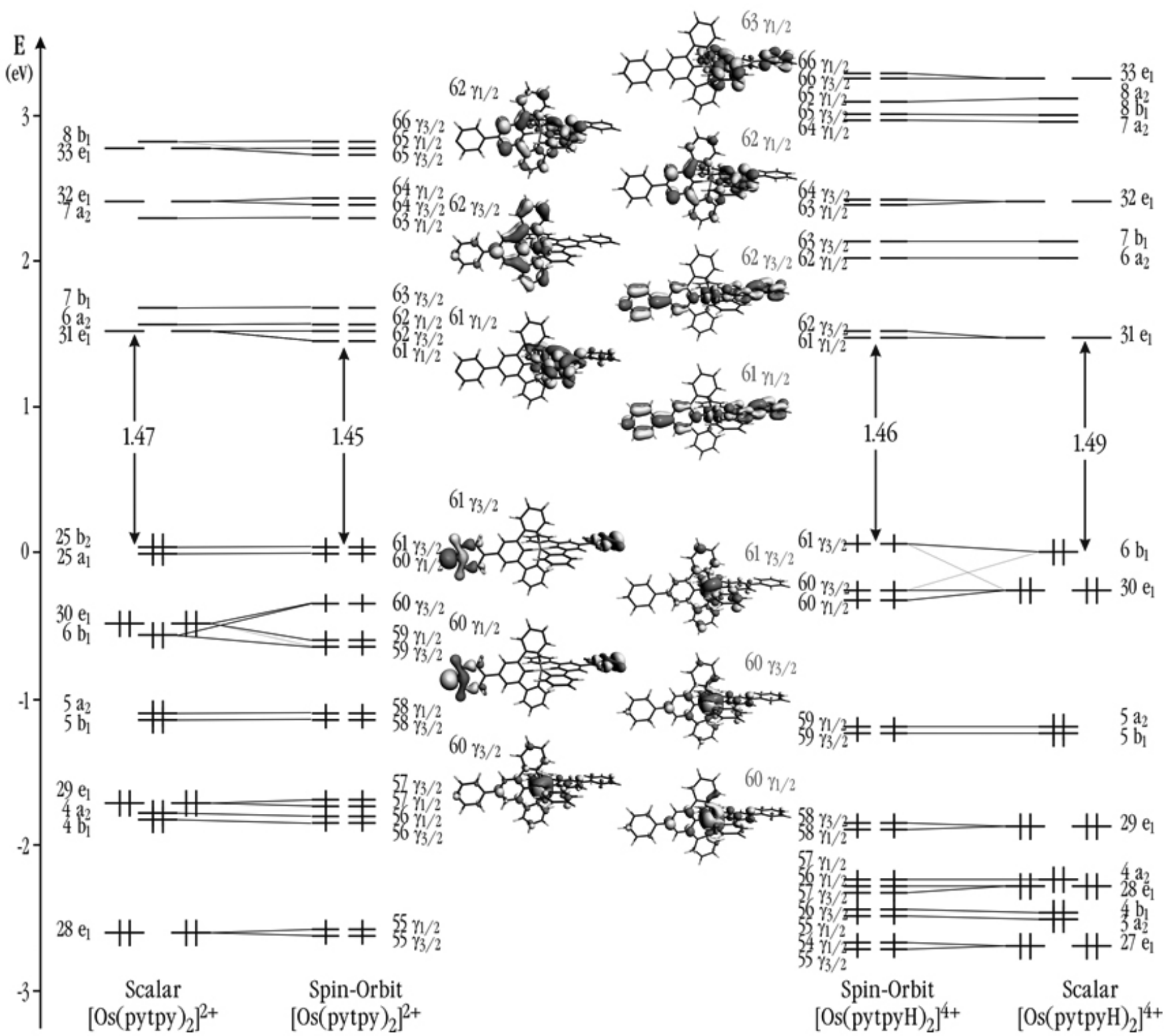

Figure 2: MS Diagram showing the correlation between the Scalar and Spin Orbit Relativistic calculations for $\left[\mathrm{Os}(\mathrm{tpy}-\mathrm{py})_{2}\right]^{2+}$ and $\left[\mathrm{Os}(\mathrm{tpy}-\mathrm{pyH})_{2}\right]^{4+}$ complexes. The MS plotted here represent the frontier MS under the Spin Orbit approach.

not induce a strong modification of the electronic structure, since the Spin Orbit coupling observed for these complexes $\left(\sim 400 \mathrm{~cm}^{-1}\right)$ is not considerable large. This small spin orbit orbit coupling occurs due to that the two fold orbitals are mainly ligand based orbitals in the case of $\left[\mathrm{Os}(\mathrm{tpy}-\mathrm{py})_{2}\right]^{2+}$. On the other hand, a large spin orbit coupling is expected for those orbitals that exhibit a large metallic character, however the two fold $e$ orbitals in $\left[\mathrm{Os}(\mathrm{tpy}-\mathrm{pyH})_{2}\right]^{4+}$ couples with the single fold orbitals of $a$ and $b$ symmetry, to give the $\gamma_{1 / 2}$ and $\gamma_{3 / 2}$ spinors at relativistic spin orbit DFT level, resulting in the small spin orbit coupling observed at Figure 2.

As can be seen from figure 2, the $60 \gamma_{1 / 2}$ and $61 \gamma_{3 / 2}$ of $[\text { Os(tpy-py) })^{2+}$ are mainly composed with the non-bonding orbitals of the free $\mathrm{N}$ electron pair which coordinates the $\mathrm{H}^{+}$ions present in $\left[\mathrm{Os}(\mathrm{tpy}-\mathrm{pyH})_{2}\right]^{4+}$ stabilizing both spinors. The importance of this fact, is that eventually, these $\mathrm{H}^{+}$could be replaced by inorganic or organometallic units [12] which can improve or modify the luminescent properties of the titled compounds.

One of the consequences of the stabilization of the $60 \gamma_{1 / 2}$ and $61 \gamma_{3 / 2}$ spinors in $\left[\mathrm{Os}(\text { tpy-py) })_{2}\right]^{2+}$ is that the frontier spinors $\left(61 \gamma_{3,2}, 60 \gamma_{1 / 2}\right.$ and $\left.60 \gamma_{3 / 2}\right)$ of the resulting $\left[\mathrm{Os}(\mathrm{tpy}-\mathrm{pyH})_{2}\right]^{4+}$ are mainly Osmium based molecular spinors, leading to a modification of the optical properties of the resulting complex, $\left[\mathrm{Os}(\text { tpy-pyH })_{2}\right]^{4+}$, compared with their parent complex $\left[\mathrm{Os}(\mathrm{tpy}-\mathrm{py})_{2}\right]^{2+}$.

To support the previous statement, we carried out time dependent density functional calculations (TD-DFT) considering the Spin Orbit relativistic effects. These results are summarized in Table 2 , were is it clearly shown that the main excitations of the $\left[\mathrm{Os}(\mathrm{tpy}-\mathrm{py})_{2}\right]^{2+}$ complex can be characterized as ligand to metal transitions (LMCT), while those of the $\left[\mathrm{Os}(\mathrm{tpy}-\mathrm{pyH})_{2}\right]^{4+}$ are typically characterized as metal to ligand transitions (MLCT), denoting the different behaviour of these complexes with respect to their optical properties. The same trend is obtained in gas phase, as well in dichloroethane COSMO modelled solvation phase, as is discussed below. 
Table 2: Excitation wavelengths (nm), Energies (eV), Oscillator strengths, Symmetry, Composition and Nature of the allowed calculated electronic transitions (dichloroethane COSMO solvation phase in Bold and gas phase in cursive).

\begin{tabular}{|c|c|c|c|c|c|c|}
\hline Compound & $\begin{array}{l}\text { Wavelength } \\
(\mathrm{nm})\end{array}$ & $\mathrm{E}(\mathrm{eV})$ & $\begin{array}{c}\text { Oscillator } \\
\text { Strength }\left(\times 10^{3}\right)\end{array}$ & Symmetry & Composition & $\begin{array}{c}\text { Nature of the } \\
\text { Electronic Transitions }\end{array}$ \\
\hline \multirow[t]{10}{*}[Os(tpy-py)_{2}]{$^{2+}$} & 746 & 1.66 & 0.335 & ${ }^{1} \mathbf{E}$ & 86.61 & $60 \gamma_{3 / 2} \rightarrow 61 \gamma_{1 / 2}$ \\
\hline & 741 & 1.67 & 0.418 & ${ }^{\prime} E$ & 88.17 & $60 \gamma_{3 / 2} \rightarrow 61 \gamma_{1 / 2}$ \\
\hline & 713 & 1.74 & 1.39 & ${ }^{1} \mathbf{E}$ & 87.87 & $60 \gamma_{3 / 2} \rightarrow 62 \gamma_{3 / 2}$ \\
\hline & 710 & 1.75 & 1.48 & ${ }^{\prime} E$ & 90.03 & $60 \gamma_{3 / 2} \rightarrow 62 \gamma_{3 / 2}$ \\
\hline & 709 & 1.75 & 19.8 & ${ }^{1} \mathbf{B}_{2}$ & 87.62 & $60 \gamma_{3 / 2} \rightarrow 61 \gamma_{1 / 2}$ \\
\hline & 700 & 1.77 & 16.5 & ${ }^{1} B_{2}$ & 83.13 & $60 \gamma_{3 / 2} \rightarrow 61 \gamma_{1 / 2}$ \\
\hline & 644 & 1.92 & 1.56 & ${ }^{1} \mathbf{E}$ & 93.88 & $60 \gamma_{3 / 2} \rightarrow 62 \gamma_{1 / 2}$ \\
\hline & 665 & 1.86 & 2.01 & ${ }^{I} E$ & 94.35 & $60 \gamma_{3 / 2} \rightarrow 62 \gamma_{1 / 2}$ \\
\hline & 634 & 1.95 & 5.67 & ${ }^{1} \mathbf{B}_{2}$ & 91.65 & $60 \gamma_{3 / 2} \rightarrow 62 \gamma_{1 / 2}$ \\
\hline & 658 & 1.88 & 8.87 & ${ }^{1} B_{2}$ & 91.31 & $60 \gamma_{3 / 2} \rightarrow 62 \gamma_{1 / 2}$ \\
\hline \multirow[t]{12}{*}[\mathrm{Os}(\mathrm{tpy}-\mathrm{pyH})_{2}]{$^{4+}$} & 825 & 1.50 & 31.94 & ${ }^{1} \mathbf{B}_{2}$ & 91.11 & $61 \gamma_{3 / 2} \rightarrow 61 \gamma_{1 / 2}$ \\
\hline & 791 & 1.57 & 0.023 & ${ }^{1} E$ & $\begin{array}{l}71.85 \\
16.33 \\
\end{array}$ & $\begin{array}{l}60 \gamma_{3 / 2} \rightarrow 61 \gamma_{1 / 2} \\
60 \gamma_{1 / 2} \rightarrow 62 \gamma_{3 / 2}\end{array}$ \\
\hline & 823 & 1.51 & 0.183 & ${ }^{1} \mathbf{E}$ & 87.30 & $61 \gamma_{3 / 2} \rightarrow 62 \gamma_{3 / 2}$ \\
\hline & 752 & 1.65 & 13.5 & ${ }^{1} B_{2}$ & $\begin{array}{l}67.11 \\
32.11 \\
\end{array}$ & $\begin{array}{l}60 \gamma_{3 / 2} \rightarrow 61 \gamma_{1 / 2} \\
60 \gamma_{1 / 2} \rightarrow 62 \gamma_{3 / 2}\end{array}$ \\
\hline & 695 & 1.78 & 8.65 & ${ }^{1} \mathbf{B}_{2}$ & \begin{tabular}{|l|}
69.59 \\
29.66 \\
\end{tabular} & $\begin{array}{l}\mathbf{6 0} \gamma_{3 / 2} \rightarrow \mathbf{6 1} \gamma_{1 / 2} \\
\mathbf{6 0} \gamma_{1 / 2} \rightarrow \mathbf{6 2} \gamma_{3 / 2}\end{array}$ \\
\hline & 634 & 1.96 & 22.04 & ${ }^{1} B_{2}$ & $\begin{array}{l}80.97 \\
13.70 \\
\end{array}$ & $\begin{array}{l}60 \gamma_{3 / 2} \rightarrow 62 \gamma_{1 / 2} \\
60 \gamma_{1 / 2} \rightarrow 62 \gamma_{3 / 2}\end{array}$ \\
\hline & 626 & 1.98 & 1.21 & ${ }^{1} \mathbf{E}$ & 97.12 & $61 \gamma_{3 / 2} \rightarrow 62 \gamma_{1 / 2}$ \\
\hline & 578 & 2.15 & 567 & ${ }^{1} B_{2}$ & $\begin{array}{l}42.47 \\
19.94 \\
14.93 \\
\end{array}$ & $\begin{array}{l}60 \gamma_{1 / 2} \rightarrow 62 \gamma_{3 / 2} \\
60 \gamma_{3 / 2} \rightarrow 61 \gamma_{1 / 2} \\
60 \gamma_{3 / 2} \rightarrow 62 \gamma_{1 / 2} \\
\end{array}$ \\
\hline & 616 & 2.01 & 2.47 & ${ }^{1} \mathbf{B}_{2}$ & 88.52 & $61 \gamma_{3 / 2} \rightarrow 62 \gamma_{1 / 2}$ \\
\hline & 548 & 2.26 & 4.12 & ${ }^{1} B_{2}$ & 88.32 & $61 \gamma_{3 / 2} \rightarrow 63 \gamma_{1 / 2}$ \\
\hline & 599 & 2.07 & 1.14 & ${ }^{1} \mathbf{E}$ & 94.71 & $61 \gamma_{3 / 2} \rightarrow 63 \gamma_{3 / 2}$ \\
\hline & 536 & 2.31 & 198 & ${ }^{1} B_{2}$ & 73.78 & $60 \gamma_{3 / 2} \rightarrow 62 \gamma_{1 / 2}$ \\
\hline
\end{tabular}

The experimental emission spectra $[4]$ shows that the $\left[\mathrm{Os}(\text { tpy-py) })^{2+}\right.$ complex exhibits an emission at $746 \mathrm{~nm}\left(\Phi_{\mathrm{em}}\left(\mathrm{x} 10^{2}\right)=0.2 ; \tau=91 \mathrm{~ns}\right)$ and the $\left[\mathrm{Os}(\text { tpy-pyH })_{2}\right]^{4+}$ complex emit at $807 \mathrm{~nm}\left(\Phi_{\mathrm{em}}\left(\mathrm{x} 10^{2}\right)=0.004 ; \tau=4 \mathrm{~ns}\right)$. Since the calculated spectral properties of Table 2 are related with the absorption experimental properties and considering that must occur a Stoke Shift through the luminiscence process, the emission behaviour could be extrapolated. Assuming these considerations, the calculations in gas phase give us the following: the $\left.[\mathrm{Os} \text { (tpy-py) })_{2}\right]^{2+}$ complex will present an emission at higher wavelengths than $710 \mathrm{~nm}$ or $741 \mathrm{~nm}$ (experimentally found at $746 \mathrm{~nm}$ ), while the $\left[\mathrm{Os}(\mathrm{tpy}-\mathrm{pyH})_{2}\right]^{4+}$ complex will present an emission at higher wavelengths than $791 \mathrm{~nm}$ (experimentally found at $807 \mathrm{~nm}$ ), as can be extracted from the calculated excitation energies presented in Table 2, expecting that the excited state shall not suffer large extent conformational changes during the optical process. On the other hand, the calculations including the dichloroethane solvation, to improve the modelling of the experimental conditions, result in a small shift for the wavelenght of the electronic excitations in the unprotonated complex compared with those of the gas phase. This small difference, does not induce any change in the electronic spectra or the emission properties of $\left.[\text { Os(tpy-py) })_{2}\right]^{2+}$ complex, as can be observed in Table 2 . With respect to the protonated complex, the inclusion of the solvent, does not modifies the electronic structure of the $\left[\mathrm{Os}(\mathrm{tpy}-\mathrm{pyH})_{2}\right]^{4+}$ complex (i.e. does not change the composition or energy of the spinors), but induces a large red shift in the wavelength of the electronic excitations, as well as a different composition of the excited states. Nevertheless, maintaining their ligand based character However, this differences induced by the solvation, are not consistent with the low experimentally obtained quantum yield and short lifetime. Constable, Housecroft et.al. [4] observed that the lifetime of excited state in the unprotonated complex is longer than the lifetime of the protonated one. This difference in the emission properties of these complexes can be explained taking into account that the excited states reached for these complexes in the absorption process are different in both complexes, while the $\left[\mathrm{Os}(\mathrm{tpy}-\mathrm{py})_{2}\right]^{2+}$ lower excited state is mainly metallic, the complex can stabilize a triplet metallic excited state, while $\left[\mathrm{Os}(\text { tpy-pyH })_{2}\right]^{4+}$ can not follow the same mechanism, since his excited state is ligand based. The later

\section{CONCLUSIONS}

The pyridine moieties are sufficiently basic to stabilize a $\mathrm{H}^{+}$, modifying the optical and luminescent properties of the $\left[\mathrm{Os}(\text { tpy-py) })_{2}\right]^{2+}$ complex, without distorting the molecular geometry. We study the effect of the protonation of $\left[\mathrm{Os}(\text { tpy-py })_{2}\right]^{2+}$ complex over the luminescent properties, concluding that the differences in their emission yields and lifetimes founded experimentally are due to the changes in the electronic structures induced by protonation and the differential composition of their lowest excited states.

\section{ACKNOWLEDGEMENTS}

The authors acknowledge the financial support of FONDECYT grants $1131123,1110758,11100027$.

\section{REFERENCES}

1.- B.L. Feringa, Molecular Switches, Wiley-VCH, Weinheim, 2001

2.- H. Dürand, H. Bouas-Laurent, Photochromism: Molecules and Systems, 
Elsevier, Amsterdam, 2003

3.- S. Silvi, E. Constable, C. Housecroft, J. Beves, E. Dunphy, M. Tomasulo, F. Raymo, A. Credi, Chem. Commun. 1484-1486 (2009)

4.- E. Constable, C. Housecroft, A. Thompson, P. Passaniti, S. Silvi, M. Maestri, A. Credi, Inorg. Chimica Acta 360, 1102-1110 (2007)

5.- Amsterdam Density Functional ADF Code, Release 2010.02, Vrije Universiteit Amsterdam, The Netherlands, 2010

6.- E. van Lenthe, E.J. Baerends, J.G. Snijders, J. Chem. Phys. 101, 97839792 (1994)
7.- G. te Velde, F.M. Bickelhaupt, S.J.A. van Gisberger, C. Fonseca, E.J. Baerends, J.G. Snijders, T.J. Ziegler, J. Comput. Chem. 22, 931-967 (2001) 8.- L. Versluis, T. Ziegler, J. Chem. Phys. 88, 322-328 (1988)

9.- J.P. Perdew, J.A. Chevary, S.H. Vosko, K.A. Jackson, M.R. Pederson, D.J. Sing and C. Fiolhais, Physical Review B 46, 6671-6687 (1992)

10.- S.J.A. van Gisbergen, J.G. Snijders, E.J. Baerends, Comput. Phys. Commun. 118, 119-138 (1999)

11.- A. Klamt, G.J. Schuurmann, J. Chem. Soc., Perkin. Trans. 2, 799-805 (1993)

12.- K. Chichak, N.R. Branda, Chem. Commun. 523-524 (1999) 